\title{
Best Linear Unbiased Prediction for Multifidelity Computer Experiments
}

\author{
Weiyan Mu (D), ${ }^{1}$ Qiuyue Wei, ${ }^{1}$ Dongli Cui, ${ }^{1}$ and Shifeng Xiong ${ }^{2}$ \\ ${ }^{1}$ School of Science, Beijing University of Civil Engineering and Architecture, Beijing 100044, China \\ ${ }^{2}$ NCMIS, Academy of Mathematics and Systems Science, Chinese Academy of Sciences, Beijing 100190, China \\ Correspondence should be addressed to Weiyan Mu; muweiyan@bucea.edu.cn
}

Received 26 September 2017; Revised 26 April 2018; Accepted 8 May 2018; Published 7 June 2018

Academic Editor: Elisa Francomano

Copyright (C) 2018 Weiyan $\mathrm{Mu}$ et al. This is an open access article distributed under the Creative Commons Attribution License, which permits unrestricted use, distribution, and reproduction in any medium, provided the original work is properly cited.

Recently it becomes a growing trend to study complex systems which contain multiple computer codes with different levels of accuracy, and a number of hierarchical Gaussian process models are proposed to handle such multiple-fidelity codes. This paper derives the best linear unbiased prediction for three popular classes of multiple-level Gaussian process models. The predictors all have explicit expressions at each untried point. Empirical best linear unbiased predictors are also provided by plug-in methods with generalized maximum likelihood estimators of unknown parameters.

\section{Introduction}

With the rapid development of computer technology, computer experiments have been widely used in engineering and science [1]. Consider a computer code with inputs $\mathbf{x} \in[0,1]^{d}$. The common statistical modeling method is to model the response of the code as

$$
Y(\mathbf{x})=\sum_{j=1}^{p} f_{j}(\mathbf{x}) \beta_{j}+Z(\mathbf{x})=f^{\prime}(\mathbf{x}) \boldsymbol{\beta}+Z(\mathbf{x}),
$$

where $f_{1}(\cdot), \ldots, f_{p}(\cdot)$ are known regression functions, $\boldsymbol{\beta}=$ $\left(\beta_{1}, \ldots, \beta_{p}\right)^{\prime}$ is a vector of unknown regression coefficients, and $Z(\cdot)$ is a stationary Gaussian process on $[0,1]^{d}$ having zero mean, variance $\sigma_{z}^{2}$, and correlation function $R(\cdot)$. Let $\mathbf{Y}=$ $\left(y_{1}, \ldots, y_{n}\right)^{\prime}$ be the responses corresponding to the design points $\mathbf{x}_{1}, \ldots, \mathbf{x}_{n} \in[0,1]^{d}$. With a known correlation function $R(\cdot)$, the best linear unbiased predictor (BLUP) is given by

$$
\widehat{Y}\left(\mathbf{x}_{\mathbf{0}}\right)=\widehat{Y}_{0} \equiv \mathbf{f}_{\mathbf{0}}{ }^{\prime} \widehat{\boldsymbol{\beta}}+\mathbf{r}_{0}^{\prime} R^{-1}\left(\mathbf{Y}^{n}-\mathbf{F} \widehat{\boldsymbol{\beta}}\right)
$$

for $\mathbf{x}_{0} \in[0,1]^{d}$, where $\mathbf{r}_{0}=\left(R\left(\mathbf{x}_{0}-\mathbf{x}_{1}\right), \ldots, R\left(\mathbf{x}_{0}-\mathbf{x}_{n}\right)\right)^{\prime}$, $\mathbf{R}=\left(R\left(\mathbf{x}_{i}-\mathbf{x}_{j}\right)\right)_{i, j=1, \ldots, n}, \mathbf{F}=\left(\mathbf{f}\left(\mathbf{x}_{1}\right), \ldots, \mathbf{f}\left(\mathbf{x}_{n}\right)\right)^{\prime}$, and $\widehat{\boldsymbol{\beta}}=$
$\left(\mathbf{F}^{\prime} \mathbf{R}^{-1} \mathbf{F}\right)^{-1} \mathbf{F}^{\prime} \mathbf{R}^{-1} \mathbf{Y}$ is the generalized least squares estimator of $\boldsymbol{\beta}$ [1].

It is a growing trend to study the complex system which contains multifidelity computer codes with different levels of accuracy. For example, a Bayesian approach is described to predict and analyze complex computer codes which can be run at different levels of sophistication [2]. A novel approach is taken to integrate data from approximate and detailed simulations to build a surrogate model that describes the relationship between output and input parameters [3]. The Bayesian hierarchical Gaussian process (BHGP) models are introduced to integrate low-accuracy and high-accuracy [4]. A class of nonstationary Gaussian process models are proposed to link the computer outputs of different mesh densities [5]. However, there are few papers that provide BLUPs for multifidelity computer experiments.

The purpose of this article is to find BLUPs for multifidelity computer experiments. The structure of the article is as follows. In Section 2, BLUPs for two levels of accuracy [4] are discussed. In Section 3, BLUPs for general $k$-level cases in autoregressive model described by Kennedy and O'Hagan [2] are illustrated. In Section 4, BLUPs for continuous level in nonstationary Gaussian process model described by Tuo, $\mathrm{Wu}$, and $\mathrm{Yu}$ [5] are demonstrated. We present a real application in Section 5. Concluding remarks are given in Section 6. 


\section{BLUPs for Two-Level Cases}

The two experiments considered in this section are named as the low-accuracy experiment (LE) and high-accuracy experiment $(\mathrm{HE})$. Let $\mathrm{GP}\left(\mu, \sigma^{2}, \boldsymbol{\theta}\right)$ denote the Gaussian process with mean $\mu$, variance $\sigma^{2}$, and correlation parameters $\boldsymbol{\theta}$. Let $D_{l}=\left(\mathbf{x}_{1}, \ldots, \mathbf{x}_{n}\right)$ and $D_{h}=\left(\mathbf{s}_{1}, \ldots, \mathbf{s}_{m}\right)$ denote the design set for the LE and HE, respectively. Following Qian and $\mathrm{Wu}$ [4], for any $\mathbf{x}_{i} \in D_{l}$, the LE is described by

$$
Y_{l}\left(\mathbf{x}_{i}\right)=\mathbf{f}^{\prime}\left(\mathbf{x}_{i}\right) \boldsymbol{\beta}+Z\left(\mathbf{x}_{i}\right),
$$

where $Z(\cdot) \sim \operatorname{GP}\left(0, \sigma_{Z}^{2}, \boldsymbol{\theta}_{1}\right)$ and $\mathbf{f}(\mathbf{x})=\left[f_{1}(\mathbf{x}), \ldots, f_{q}(\mathbf{x})\right]^{\prime}$ is a set of prespecified regression functions. For any $\mathbf{s}_{i} \in D_{h}$, the HE can be described by

$$
Y_{h}\left(\mathbf{s}_{i}\right)=\rho\left(\mathbf{s}_{i}\right) y_{l}\left(\mathbf{s}_{i}\right)+\delta\left(\mathbf{s}_{i}\right)+\varepsilon\left(\mathbf{s}_{i}\right),
$$

where the scale changes from LE to $\operatorname{HE} \rho(\cdot) \sim \operatorname{GP}\left(\rho_{0}, \sigma_{\rho}^{2}, \boldsymbol{\theta}_{3}\right)$, the location adjustment $\delta(\cdot) \sim \mathrm{GP}\left(\delta_{0}, \sigma_{\delta}^{2}, \boldsymbol{\theta}_{2}\right)$, the measurement error $\varepsilon \sim N\left(0, \sigma_{\varepsilon}^{2}\right)$ and $Z(\cdot), \delta(\cdot)$, and $\varepsilon$ are jointly independent. Let $\mathbf{Y}_{l}=\left(Y_{l}\left(\mathbf{x}_{1}\right), \ldots, Y_{l}\left(\mathbf{x}_{n}\right)\right)^{\prime}$ and $\mathbf{Y}_{h}=\left(Y_{l}\left(\mathbf{s}_{1}\right), \ldots\right.$, $\left.Y_{l}\left(\mathbf{s}_{m}\right)\right)^{\prime}$.

Theorem 1. For $\mathbf{x}_{0} \in[0,1]^{d}$, the BLUP of $Y_{h}\left(\mathbf{x}_{0}\right)$ is $\mathbf{a}^{\prime} \mathbf{Y}$, where

a

$$
\begin{aligned}
& =\mathbf{B}^{-1} \mathbf{A}\left(\mathbf{A}^{\prime} \mathbf{B}^{-1} \mathbf{A}\right)^{-1} \mathbf{v}_{1}+\left[\mathbf{B}^{-1}-\mathbf{B}^{-1} \mathbf{A}\left(\mathbf{A}^{\prime} \mathbf{B}^{-1} \mathbf{A}\right)^{-1} \mathbf{A}^{\prime} \mathbf{B}^{-1}\right] \mathbf{v}_{2}, \\
& \mathbf{A}=\left[\begin{array}{cc}
\mathbf{F}_{1} & \mathbf{0} \\
\rho_{0} \mathbf{F}_{2} & \mathbf{1}_{m}
\end{array}\right], \\
& \mathbf{v}_{1}=\left[\begin{array}{cc}
\rho_{0} \mathbf{f}\left(\mathbf{x}_{0}\right) \\
1
\end{array}\right], \\
& \mathbf{v}_{2}=\left[\begin{array}{c}
\rho_{0} \sigma_{Z}^{2} \mathbf{r}_{\boldsymbol{\theta}_{1}}^{1} \\
\sigma_{\rho}^{2} \mathbf{v} \boldsymbol{\beta} \boldsymbol{\beta}^{\prime} \mathbf{f}\left(\mathbf{x}_{0}\right)+\sigma_{\rho}^{2} \sigma_{Z}^{2} \mathbf{v}_{1}+\rho_{0}^{2} \sigma_{Z}^{2} \mathbf{r}_{\boldsymbol{\theta}_{1}}^{2}+\sigma_{\delta}^{2} \mathbf{r}_{\theta_{2}}
\end{array}\right],
\end{aligned}
$$

and $\mathbf{B}$

$$
=\left[\begin{array}{cc}
\sigma_{Z}^{2} \mathbf{R}_{\boldsymbol{\theta}_{1}}^{1} & \rho_{0} \sigma_{Z}^{2} \mathbf{R}_{\boldsymbol{\theta}_{1}}^{3} \\
\rho_{0} \sigma_{Z}^{2} \mathbf{R}_{\boldsymbol{\theta}_{1}}^{3}{ }^{\prime} & \sigma_{\rho}^{2} \boldsymbol{\beta}^{\prime} \mathbf{V} \boldsymbol{\beta}+\sigma_{\rho}^{2} \sigma_{Z}^{2} \mathbf{V}_{1}+\rho_{0}^{2} \sigma_{Z}^{2} \mathbf{R}_{\boldsymbol{\theta}_{1}}^{2}+\sigma_{\delta}^{2} \mathbf{R}_{\boldsymbol{\theta}_{2}}+\sigma_{\epsilon}^{2} \mathbf{I}_{m}
\end{array}\right] .
$$

Proof. The linear predictor $\widehat{Y}_{h}\left(\mathbf{x}_{0}\right)=\mathbf{a}_{1}^{\prime} \mathbf{Y}_{l}+\mathbf{a}_{2}^{\prime} \mathbf{Y}_{h}+C$ based on training data $\mathbf{Y}_{L}$ and $\mathbf{Y}_{H}$ at an untried point $\mathbf{x}_{0}$ is unbiased for $Y_{h}\left(\mathbf{x}_{0}\right)$ provided

$$
\begin{aligned}
\mathbf{a}_{1}^{\prime} \mathbf{F}_{1}+\rho_{0} \mathbf{a}_{2}^{\prime} \mathbf{F}_{2} & =\rho_{0} \mathbf{f}^{\prime}\left(\mathbf{x}_{0}\right), \\
\mathbf{a}_{2}^{\prime} \mathbf{1}_{m} & =1, \\
C & =0 .
\end{aligned}
$$

For any linear unbiased predictor (LUP) of $Y_{0}=Y_{h}\left(\mathbf{x}_{0}\right)$, $\widehat{Y}_{h}\left(\mathbf{x}_{0}\right)=\mathbf{a}_{1}^{\prime} \mathbf{Y}_{l}+\mathbf{a}_{2}^{\prime} \mathbf{Y}_{h}$, the mean squared prediction error (MSPE) of $\mathbf{a}_{1}^{\prime} \mathbf{Y}_{l}+\mathbf{a}_{2}^{\prime} \mathbf{Y}_{h}$ is $E\left\{M^{2}\right\}=E\left\{\left(\mathbf{a}_{1}^{\prime} \mathbf{Y}_{l}+\mathbf{a}_{2}^{\prime} \mathbf{Y}_{h}-Y_{0}\right)^{2}\right\}$.

We have

$$
\begin{aligned}
& E\left\{M^{2}\right\} \\
& =\sigma_{\rho}^{2}\left(\mathbf{a}_{2}^{\prime} \boldsymbol{\beta}^{\prime} \mathbf{V} \boldsymbol{\beta} \mathbf{a}_{2}+\mathbf{f}^{\prime}\left(\mathbf{x}_{0}\right) \boldsymbol{\beta} \boldsymbol{\beta}^{\prime} \mathbf{f}\left(\mathbf{x}_{0}\right)-2 \mathbf{a}_{2}^{\prime} \mathbf{v} \boldsymbol{\beta} \boldsymbol{\beta}^{\prime} \mathbf{f}\left(\mathbf{x}_{0}\right)\right)
\end{aligned}
$$

$$
\begin{aligned}
& +\sigma_{Z}^{2} \mathbf{a}_{1}^{\prime} \mathbf{R}_{\boldsymbol{\theta}_{1}}^{1} \mathbf{a}_{1}+\sigma_{Z}^{2} \sigma_{\rho}^{2} \mathbf{a}_{2}^{\prime} \mathbf{V}_{1} \mathbf{a}_{2}+\rho_{0}^{2} \sigma_{Z}^{2} \mathbf{a}_{2}^{\prime} \mathbf{R}_{\boldsymbol{\theta}_{1}}^{2} \mathbf{a}_{2} \\
& +\left(\sigma_{\rho}^{2}+\rho_{0}^{2}\right) \sigma_{Z}^{2}+2 \rho_{0} \sigma_{Z}^{2} \mathbf{a}_{1}^{\prime} \mathbf{R}_{\boldsymbol{\theta}_{1}}^{3} \mathbf{a}_{2}-2 \rho_{0} \sigma_{Z}^{2} \mathbf{a}_{1}^{\prime} \mathbf{r}_{\boldsymbol{\theta}_{1}}^{1} \\
& -2 \sigma_{Z}^{2} \sigma_{\rho}^{2} \mathbf{a}_{2}^{\prime} \mathbf{v}_{1}-2 \rho_{0}^{2} \sigma_{Z}^{2} \mathbf{a}_{2}^{\prime} \mathbf{r}_{\boldsymbol{\theta}_{1}}^{2} \\
& +\sigma_{\delta}^{2}\left(\mathbf{a}_{2}^{\prime} \mathbf{R}_{\boldsymbol{\theta}_{2}} \mathbf{a}_{2}-2 \mathbf{a}_{2}^{\prime} \mathbf{r}_{\boldsymbol{\theta}_{2}}+1\right)+\sigma_{\epsilon}^{2}\left(\mathbf{a}_{2}^{\prime} \mathbf{a}_{2}+1\right),
\end{aligned}
$$

where $\mathbf{V}=\left(R_{\boldsymbol{\theta}_{3}}\left(\mathbf{s}_{i}-\mathbf{s}_{j}\right) \mathbf{f}\left(\mathbf{s}_{i}\right) \mathbf{f}^{\prime}\left(\mathbf{s}_{j}\right)\right), \mathbf{V}_{1}=\left(R_{\boldsymbol{\theta}_{1}}\left(\mathbf{s}_{i}-\mathbf{s}_{j}\right) R_{\boldsymbol{\theta}_{3}}\left(\mathbf{s}_{i}-\right.\right.$ $\left.\left.\mathbf{s}_{j}\right)\right), \mathbf{R}_{\boldsymbol{\theta}_{1}}^{2}=\left(R_{\boldsymbol{\theta}_{1}}\left(\mathbf{s}_{i}-\mathbf{s}_{j}\right)\right), \mathbf{R}_{\boldsymbol{\theta}_{2}}=\left(R_{\boldsymbol{\theta}_{2}}\left(\mathbf{s}_{i}-\mathbf{s}_{j}\right)\right), 1 \leq i, j \leq m$; $\mathbf{v}=\left(R_{\boldsymbol{\theta}_{3}}\left(\mathbf{x}_{0}-\mathbf{s}_{i}\right) \mathbf{f}^{\prime}\left(\mathbf{s}_{i}\right)\right), \mathbf{v}_{1}=\left(R_{\boldsymbol{\theta}_{1}}\left(\mathbf{x}_{0}-\mathbf{s}_{i}\right) R_{\boldsymbol{\theta}_{3}}\left(\mathbf{x}_{0}-\mathbf{s}_{i}\right)\right), \mathbf{r}_{\boldsymbol{\theta}_{1}}^{2}=$ $\left(R_{\boldsymbol{\theta}_{1}}\left(\mathbf{x}_{0}-\mathbf{s}_{i}\right)\right)^{\prime}, \mathbf{r}_{\boldsymbol{\theta}_{2}}=\left(R_{\boldsymbol{\theta}_{2}}\left(\mathbf{x}_{0}-\mathbf{s}_{i}\right)\right)^{\prime}, 1 \leq i \leq m ; \mathbf{R}_{\boldsymbol{\theta}_{1}}^{1}=\left(R_{\boldsymbol{\theta}_{1}}\left(\mathbf{x}_{i}-\right.\right.$ $\left.\left.\mathbf{x}_{j}\right)\right), 1 \leq i, j \leq n ; \mathbf{R}_{\boldsymbol{\theta}_{1}}^{3}=\left(R_{\boldsymbol{\theta}_{1}}\left(\mathbf{x}_{i}-\mathbf{s}_{j}\right)\right), 1 \leq i \leq m, 1 \leq j \leq n$; $\mathbf{r}_{\boldsymbol{\theta}_{1}}^{1}=\left(R_{\boldsymbol{\theta}_{1}}\left(\mathbf{x}_{0}-\mathbf{x}_{i}\right)\right)^{\prime}, 1 \leq i \leq n$.

Thus the Lagrange multipliers can be used to solve BLUP corresponding to $\mathbf{a}_{1}$ and $\mathbf{a}_{2}$ that minimize (5) subject to

$$
\begin{aligned}
\mathbf{a}_{1}^{\prime} \mathbf{F}_{1}+\rho_{0} \mathbf{a}_{2}^{\prime} \mathbf{F}_{2} & =\rho_{0} \mathbf{f}^{\prime}\left(\mathbf{x}_{0}\right), \\
\mathbf{a}_{2}^{\prime} \mathbf{1}_{m} & =1 .
\end{aligned}
$$

The Lagrange function is

$$
\begin{aligned}
L & =\sigma_{\rho}^{2}\left(\mathbf{a}_{2}^{\prime} \boldsymbol{\beta}^{\prime} \mathbf{V} \boldsymbol{\beta} \mathbf{a}_{2}+\mathbf{f}^{\prime}\left(\mathbf{x}_{0}\right) \boldsymbol{\beta} \boldsymbol{\beta}^{\prime} \mathbf{f}\left(\mathbf{x}_{0}\right)\right. \\
& \left.-2 \mathbf{a}_{2}^{\prime} \mathbf{v} \boldsymbol{\beta} \boldsymbol{\beta}^{\prime} \mathbf{f}\left(\mathbf{x}_{0}\right)\right)+\sigma_{Z}^{2} \mathbf{a}_{1}^{\prime} \mathbf{R}_{\boldsymbol{\theta}_{1}}^{1} \mathbf{a}_{1} \sigma_{Z}^{2} \sigma_{\rho}^{2} \mathbf{a}_{2}^{\prime} \mathbf{V}_{1} \mathbf{a}_{2} \\
& +\rho_{0}^{2} \sigma_{Z}^{2} \mathbf{a}_{2}^{\prime} \mathbf{R}_{\boldsymbol{\theta}_{1}}^{2} \mathbf{a}_{2}+\left(\sigma_{\rho}^{2}+\rho_{0}^{2}\right) \sigma_{Z}^{2}+2 \rho_{0} \sigma_{Z}^{2} \mathbf{a}_{1}^{\prime} \mathbf{R}_{\boldsymbol{\theta}_{1}}^{3} \mathbf{a}_{2} \\
& -2 \rho_{0} \sigma_{Z}^{2} \mathbf{a}_{1}^{\prime} \mathbf{r}_{\boldsymbol{\theta}_{1}}^{1}-2 \sigma_{Z}^{2} \sigma_{\rho}^{2} \mathbf{a}_{2}^{\prime} \mathbf{v}_{1}-2 \rho_{0}^{2} \sigma_{Z}^{2} \mathbf{a}_{2}^{\prime} \mathbf{r}_{\boldsymbol{\theta}_{1}}^{2} \\
& +\sigma_{\delta}^{2}\left(\mathbf{a}_{2}^{\prime} \mathbf{R}_{\boldsymbol{\theta}_{2}} \mathbf{a}_{2}-2 \mathbf{a}_{2}^{\prime} \mathbf{r}_{\boldsymbol{\theta}_{2}}+1\right)+\sigma_{\epsilon}^{2}\left(\mathbf{a}_{2}^{\prime} \mathbf{a}_{2}+1\right) \\
& +2 \lambda_{1}^{\prime}\left(\mathbf{F}_{1}^{\prime} \mathbf{a}_{1}+\rho_{0} \mathbf{F}_{2}^{\prime} \mathbf{a}_{2}-\rho_{0} \mathbf{f}\left(\mathbf{x}_{\mathbf{0}}\right)\right)+2 \lambda_{2}\left(\mathbf{1}_{m}^{\prime} \mathbf{a}_{2}\right. \\
& -1)
\end{aligned}
$$

Let the gradient with respect to $\mathbf{a}_{1}, \mathbf{a}_{2}, \lambda_{1}, \lambda_{2}$ be zero, and we have

$$
\left[\begin{array}{ll}
\mathbf{0} & \mathbf{A}^{\prime} \\
\mathbf{A} & \mathbf{B}
\end{array}\right]\left[\begin{array}{l}
\lambda \\
\mathbf{a}
\end{array}\right]=\left[\begin{array}{l}
\mathbf{v}_{1} \\
\mathbf{v}_{2}
\end{array}\right]
$$

where

$$
\begin{aligned}
& \mathbf{A}=\left[\begin{array}{cc}
\mathbf{F}_{1} & \mathbf{0} \\
\rho_{0} \mathbf{F}_{2} & \mathbf{1}_{m}
\end{array}\right] \\
& \mathbf{v}_{1}=\left[\begin{array}{c}
\rho_{0} \mathbf{f}\left(\mathbf{x}_{0}\right) \\
1
\end{array}\right] \\
& \mathbf{v}_{2}=\left[\begin{array}{c}
\rho_{0} \sigma_{Z}^{2} \mathbf{r}_{\boldsymbol{\theta}_{1}}^{1} \\
\sigma_{\rho}^{2} \mathbf{v} \boldsymbol{\beta} \boldsymbol{\beta}^{\prime} \mathbf{f}\left(\mathbf{x}_{0}\right)+\sigma_{\rho}^{2} \sigma_{Z}^{2} \mathbf{v}_{1}+\rho_{0}^{2} \sigma_{Z}^{2} \mathbf{r}_{\boldsymbol{\theta}_{1}}^{2}+\sigma_{\delta}^{2} \mathbf{r}_{\boldsymbol{\theta}_{2}}
\end{array}\right]
\end{aligned}
$$

B

$$
=\left[\begin{array}{cc}
\sigma_{Z}^{2} \mathbf{R}_{\boldsymbol{\theta}_{1}}^{1} & \rho_{0} \sigma_{Z}^{2} \mathbf{R}_{\boldsymbol{\theta}_{1}}^{3} \\
\rho_{0} \sigma_{Z}^{2} \mathbf{R}_{\boldsymbol{\theta}_{1}}^{3{ }^{\prime}} & \sigma_{\rho}^{2} \boldsymbol{\beta}^{\prime} \mathbf{V} \boldsymbol{\beta}+\sigma_{\rho}^{2} \sigma_{Z}^{2} \mathbf{V}_{1}+\rho_{0}^{2} \sigma_{Z}^{2} \mathbf{R}_{\boldsymbol{\theta}_{1}}^{2}+\sigma_{\delta}^{2} \mathbf{R}_{\boldsymbol{\theta}_{2}}+\sigma_{\epsilon}^{2} \mathbf{I}_{m}
\end{array}\right] .
$$

After a small amount of algebra, the a solution gives

$$
\mathbf{a}=\mathbf{B}^{-1} \mathbf{A}\left(\mathbf{A}^{\prime} \mathbf{B}^{-1} \mathbf{A}\right)^{-1} \mathbf{v}_{1}
$$




$$
+\left[\mathbf{B}^{-1}-\mathbf{B}^{-1} \mathbf{A}\left(\mathbf{A}^{\prime} \mathbf{B}^{-1} \mathbf{A}\right)^{-1} \mathbf{A}^{\prime} \mathbf{B}^{-1}\right] \mathbf{v}_{2}
$$

Thus the BLUP of $Y_{h}\left(\mathbf{x}_{0}\right)$, the computer output at an untried point $\mathbf{x}_{0}$, is $\mathbf{a}^{\prime} \mathbf{Y}$.

Note that the parameters $\beta$ and $\rho_{0}$ are often unknown in (10). We can use their estimators to replace them and get the empirical BLUP. For $\boldsymbol{\beta}$, we use its maximum likelihood estimate (MLE) based on the data $\mathbf{Y}_{L}=\mathbf{F}_{1} \boldsymbol{\beta}+\mathbf{Z}_{1}$,

$$
\widehat{\boldsymbol{\beta}}=\left(\mathbf{F}_{1}^{\prime}\left(\mathbf{R}_{\theta_{1}}^{1}\right)^{-1} \mathbf{F}_{1}\right)^{-1} \mathbf{F}_{1}^{\prime}\left(\mathbf{R}_{\theta_{1}}^{1}\right)^{-1} \mathbf{Y}_{L}
$$

To estimate $\rho_{0}$, we first predict the computer output at $D_{H}=\left(\mathbf{s}_{1}, \ldots, \mathbf{s}_{m}\right)$, say $\widehat{\mathbf{Y}}_{L}^{*}$, based on the assumption $Z_{1}(\cdot) \sim$ $\mathrm{GP}\left(\mathbf{h}^{\prime}(\cdot) \boldsymbol{\beta}_{1}, \sigma_{1}^{2}, b_{1}\right)$ and training data $\left(D_{1}, \mathbf{Z}_{1}\right)$. We then estimate by MLE based on the model $\mathbf{Y}_{h}\left(\mathbf{x}_{i}\right)=\rho_{0} \widehat{\mathbf{Y}}_{l}\left(\mathbf{x}_{i}\right)+\delta\left(\mathbf{x}_{i}\right)+$ $\epsilon\left(\mathbf{x}_{i}\right)$.

The log likelihood, up to an additive constant, can be written as

$$
\begin{aligned}
- & \frac{1}{2}\left(\mathbf{Y}_{H}-\rho_{0} \widehat{\mathbf{Y}}_{L}^{*}-\delta_{0} \mathbf{1}_{m}\right)^{\prime}\left(\sigma_{\rho}^{2} \mathbf{V}+\sigma_{\delta}^{2} \mathbf{R}_{\theta_{2}}+\sigma_{\epsilon}^{2} \mathbf{I}_{m}\right)^{-1} \\
& \cdot\left(\mathbf{Y}_{H}-\rho_{0} \widehat{\mathbf{Y}}_{L}^{*}-\delta_{0} \mathbf{1}_{m}\right),
\end{aligned}
$$

where

$$
\mathbf{V}=\left(\begin{array}{cccc}
\widehat{Y}_{l 1}^{2} & R_{\theta_{3}}\left(\mathbf{s}_{1}-\mathbf{s}_{2}\right) \widehat{Y}_{l 1} \widehat{Y}_{l 2} & \cdots & R_{\theta_{3}}\left(\mathbf{s}_{1}-\mathbf{s}_{m}\right) \widehat{Y}_{l 1} \widehat{Y}_{l m} \\
R_{\theta_{3}}\left(\mathbf{s}_{1}-\mathbf{s}_{2}\right) \widehat{Y}_{l 1} \widehat{Y}_{l 2} & \widehat{Y}_{l 2}^{2} & \cdots & R_{\theta_{3}}\left(\mathbf{s}_{2}-\mathbf{s}_{m}\right) \widehat{Y}_{l 2} \widehat{Y}_{l m} \\
\vdots & \vdots & & \vdots \\
R_{\theta_{3}}\left(\mathbf{s}_{1}-\mathbf{s}_{m}\right) \widehat{Y}_{l 1} \widehat{Y}_{l m} & R_{\theta_{3}}\left(\mathbf{s}_{2}-\mathbf{s}_{m}\right) \widehat{Y}_{l 2} \widehat{Y}_{l m} & \cdots & \widehat{Y}_{l m}^{2}
\end{array}\right),
$$

and then the MLE of $\rho_{0}$ is

$$
\begin{aligned}
\widehat{\rho}_{0}= & \mathbf{Q}^{-1} \widehat{\mathbf{Y}}_{L}^{*}\left(\sigma_{\rho}^{2} \mathbf{V}+\sigma_{\delta}^{2} \mathbf{R}_{\theta_{2}}+\sigma_{\epsilon}^{2} \mathbf{I}_{m}\right)^{-1} \mathbf{Y}_{H} \\
& -\mathbf{Q}^{-1} \mathbf{A}_{1}^{\prime} \mathbf{B}_{1}^{-1} \mathbf{1}_{m}^{\prime}\left(\sigma_{\rho}^{2} \mathbf{V}+\sigma_{\delta}^{2} \mathbf{R}_{\theta_{2}}+\sigma_{\epsilon}^{2} \mathbf{I}_{m}\right)^{-1} \mathbf{Y}_{H}
\end{aligned}
$$

\section{BLUPs for $m$-Level Cases $(k>2)$}

For each $t=1, \ldots, k, D_{t}$ denotes the design set consisting of the $n_{t}$ points $\mathbf{x}_{1}^{(t)}, \ldots, \mathbf{x}_{n_{t}}^{(t)}$ and $\mathbf{Z}^{\prime}=\left(\mathbf{Z}_{1}^{\prime}, \ldots, \mathbf{Z}_{k}^{\prime}\right)$ denotes the output data, where $\mathbf{Z}_{t}^{\prime}=\left(Z_{t}\left(\mathbf{x}_{1}^{(t)}\right), \ldots, Z_{t}\left(\mathbf{x}_{n_{t}}^{(t)}\right)\right)$ is the vector of outputs for the level $t$ code.

The autoregressive model [2] can be described by

$$
Z_{t}(\mathbf{x})=\rho_{t-1} Z_{t-1}(\mathbf{x})+\delta_{t}(\mathbf{x}) \quad(t=2, \ldots, k),
$$

where $Z_{1} \sim \mathrm{GP}\left(\mathbf{h}(\cdot)^{\prime} \boldsymbol{\beta}_{1}, \sigma_{1}^{2}, b_{1}\right), \delta_{t} \sim \mathrm{GP}\left(\mathbf{h}(\cdot)^{\prime} \boldsymbol{\beta}_{t}, \sigma_{t}^{2}, b_{t}\right)$, and $\delta_{t}(\cdot)$ is independent of $Z_{t-1}(\cdot), \ldots, Z_{1}(\cdot)$.

Theorem 2. The BLUP of $Z_{k}\left(x_{0}\right)$ is $\mathbf{a}^{\prime} \mathbf{Z}$, where

$$
\begin{aligned}
\mathbf{a}= & \mathbf{Q}^{-1} \mathbf{H}\left(\mathbf{H}^{\prime} \mathbf{Q}^{-1} \mathbf{H}\right)^{-1} \mathbf{h} \\
& +\left[\mathbf{Q}^{-1}-\mathbf{Q}^{-1} \mathbf{H}\left(\mathbf{H}^{\prime} \mathbf{Q}^{-1} \mathbf{H}\right)^{-1} \mathbf{H}^{\prime} \mathbf{Q}^{-1}\right] \mathbf{V}_{2}
\end{aligned}
$$

where the notation is given in the proof.

Proof. The linear predictor $\mathbf{a}^{\prime} \mathbf{Z}+C$ is unbiased for $Z_{s}\left(\mathbf{x}_{0}\right)$ provided

$$
\begin{aligned}
\mathbf{a}^{\prime} \mathbf{H} & =\mathbf{h}^{\prime}, \\
C & =0 .
\end{aligned}
$$

where

$$
\mathbf{a}=\left(\begin{array}{c}
\mathbf{a}_{1} \\
\mathbf{a}_{2} \\
\vdots \\
\mathbf{a}_{k}
\end{array}\right)
$$$$
\mathbf{h}=\left(\begin{array}{c}
\rho_{1} \cdots \rho_{s-1} \mathbf{h}\left(\mathbf{x}_{0}\right) \\
\rho_{2} \cdots \rho_{s-1} \mathbf{h}\left(\mathbf{x}_{0}\right) \\
\vdots \\
\left.\mathbf{h}\left(\mathbf{x}_{0}\right)^{\prime}\right)
\end{array}\right)
$$$$
\mathbf{H}_{t}=\left(\begin{array}{c}
\mathbf{h}\left(\mathbf{x}_{1}^{(t)}\right) \\
\mathbf{h}\left(\mathbf{x}_{2}^{(t)}\right) \\
\vdots \\
\mathbf{h}\left(\mathbf{x}_{n_{t}}^{(t)}\right)
\end{array}\right),
$$

$$
\mathbf{H}=\left(\begin{array}{cccc}
\mathbf{H}_{1} & \mathbf{0} & \cdots & \mathbf{0} \\
\rho_{1} \mathbf{H}_{2} & \mathbf{H}_{2} & \cdots & \mathbf{0} \\
\vdots & \vdots & & \vdots \\
\rho_{1} \cdots \rho_{k-1} \mathbf{H}_{k} & \rho_{2} \cdots \rho_{s-1} \mathbf{H}_{s} & \cdots & \mathbf{H}_{k}
\end{array}\right) .
$$

For any LUP of $\widehat{Z}_{k}\left(\mathbf{x}_{0}\right)$, say $\mathbf{a}^{\prime} \mathbf{Z}$, the MSPE of $\mathbf{a}^{\prime} \mathbf{Z}$ is

$$
E\left[M^{2}\right]=E\left[\mathbf{a}_{1}^{\prime} \mathbf{Z}_{1}+\mathbf{a}_{2}^{\prime} \mathbf{Z}_{2}+\cdots+\left(\mathbf{a}_{k}^{\prime} \mathbf{Z}_{k}-Z_{s}\left(\mathbf{x}_{0}\right)\right)\right]^{2}
$$




$$
\begin{aligned}
= & \sum_{j=1}^{k} \mathbf{a}_{j}^{\prime} \mathbf{V}_{(j)} \mathbf{a}_{j}+V_{0}-2 \sum_{j=1}^{k} \mathbf{a}_{j}^{\prime} \mathbf{V}_{(j, 0)} \\
& +\sum_{j=1}^{k-1} \sum_{l=j+1}^{k} \mathbf{a}_{j}^{\prime} \mathbf{V}_{(j, l)} \mathbf{a}_{l}+\sum_{j=1}^{k-1} \sum_{l=j+1}^{k} \mathbf{a}_{l}^{\prime} \mathbf{V}_{(l, j)} \mathbf{a}_{j} .
\end{aligned}
$$

Let $\mathbf{A}_{t}\left(D_{j}, D_{l}\right)$ denote the matrix of correlations between points in $D_{j}$ and $D_{l} ; \mathbf{A}_{t}\left(D_{j}\right)$ is the abbreviation for $\mathbf{A}_{t}\left(D_{j}, D_{j}\right)$. The notation in (22) is as follows:

$$
\begin{aligned}
& \mathbf{V}_{(j)}=\sigma_{k}^{2} \mathbf{A}_{j}\left(D_{j}\right)+\rho_{j-1}^{2} \sigma_{j-1}^{2} \mathbf{A}_{j-1}\left(D_{j}\right) \\
& +\rho_{j-1}^{2} \rho_{j-2}^{2} \sigma_{j-2}^{2} \mathbf{A}_{j-2}\left(D_{j}\right)+\cdots \\
& +\left(\rho_{1} \cdots \rho_{j-1}\right)^{2} \sigma_{1}^{2} \mathbf{A}_{1}\left(D_{j}\right) \\
& V_{0}=\sigma_{k}^{2}+\rho_{k-1}^{2} \sigma_{k-1}^{2}+\rho_{k-1}^{2} \rho_{k-2}^{2} \sigma_{k-2}^{2}+\cdots \\
& +\left(\rho_{1} \cdots \rho_{k-1}\right)^{2} \sigma_{1}^{2} \\
& \mathbf{V}_{(j, l)}=\left(\rho_{j} \cdots \rho_{l-1}\right) \sigma_{j}^{2} \mathbf{A}_{j}\left(D_{j}, D_{l}\right) \\
& +\left(\rho_{j-1} \cdots \rho_{l-1}\right) \rho_{j-1} \sigma_{j-1}^{2} \mathbf{A}_{j-1}\left(D_{j}, D_{l}\right) \\
& +\left(\rho_{j-2} \cdots \rho_{l-1}\right) \rho_{j-2} \rho_{j-1} \sigma_{j-2}^{2} \mathbf{A}_{j-2}\left(D_{j}, D_{l}\right) \\
& +\cdots \\
& +\left(\rho_{1} \cdots \rho_{j-1}\right)\left(\rho_{1} \cdots \rho_{l-1}\right) \sigma_{1}^{2} \mathbf{A}_{1}\left(D_{j}, D_{l}\right) ; \\
& \mathbf{V}_{(j, 0)}=\left(\rho_{1} \cdots \rho_{k-1}\right)\left(\rho_{1} \cdots \rho_{j-1}\right) \sigma_{1}^{2} \mathbf{A}_{1}\left(D_{j}, \mathbf{x}_{0}\right) \\
& +\left(\rho_{2} \cdots \rho_{k-1}\right)\left(\rho_{2} \cdots \rho_{j-1}\right) \sigma_{2}^{2} \mathbf{A}_{2}\left(D_{j}, \mathbf{x}_{0}\right) \\
& +\cdots \\
& +\left(\rho_{j-1}\right)\left(\rho_{j-1} \cdots \rho_{k-1}\right) \sigma_{j-1}^{2} \mathbf{A}_{j-1}\left(D_{j}, \mathbf{x}_{0}\right) \\
& +\left(\rho_{j} \cdots \rho_{k-1}\right) \sigma_{j}^{2} \mathbf{A}_{k}\left(D_{k}, \mathbf{x}_{0}\right) .
\end{aligned}
$$

The Lagrange function is

$$
\begin{aligned}
L= & \sum_{j=1}^{k} \mathbf{a}_{j}^{\prime} \mathbf{V}_{(j)} \mathbf{a}_{j}-2 \sum_{j=1}^{k} \mathbf{a}_{j}^{\prime} \mathbf{V}_{(j, 0)}+\sum_{j=1}^{k-1} \sum_{l=j+1}^{k} \mathbf{a}_{j}^{\prime} \mathbf{V}_{(j, l)} \mathbf{a}_{l} \\
& +\sum_{j=1}^{k-1} \sum_{l=j+1}^{k} \mathbf{a}_{l}^{\prime} \mathbf{V}_{(l, j)} \mathbf{a}_{j}+V_{0}+2 \lambda^{\prime}\left(\mathbf{H}^{\prime} \mathbf{a}-\mathbf{h}\right) \\
= & \mathbf{a}^{\prime} \mathbf{V}_{1} \mathbf{a}-2 \mathbf{a}^{\prime} \mathbf{V}_{2}+\mathbf{a}^{\prime} \mathbf{V}_{3} \mathbf{a}+\mathbf{a}^{\prime} \mathbf{V}_{4} \mathbf{a} \\
& +2 \lambda^{\prime}\left(\mathbf{H}^{\prime} \mathbf{a}-\mathbf{h}\right),
\end{aligned}
$$

where

$$
\mathbf{V}_{1}=\left(\begin{array}{cccc}
\mathbf{V}_{(1)} & \mathbf{0} & \cdots & \mathbf{0} \\
\mathbf{0} & \mathbf{V}_{(2)} & \cdots & \mathbf{0} \\
\vdots & \vdots & & \vdots \\
\mathbf{0} & \mathbf{0} & \cdots & \mathbf{V}_{(k)}
\end{array}\right)
$$$$
\mathbf{V}_{2}=\left(\begin{array}{c}
\mathbf{V}_{(1,0)} \\
\mathbf{V}_{(2,0)} \\
\vdots \\
\mathbf{V}_{(k, 0)}
\end{array}\right) \text {, }
$$$$
\mathbf{V}_{3}=\left(\begin{array}{cccc}
\mathbf{0} & \mathbf{V}_{(1,2)} & \cdots & \mathbf{V}_{(1, k)} \\
\mathbf{0} & \mathbf{0} & \cdots & \mathbf{V}_{(2, k)} \\
\vdots & \vdots & & \vdots \\
\mathbf{0} & \mathbf{0} & \cdots & \mathbf{0}
\end{array}\right) \text {, }
$$$$
\mathbf{V}_{4}=\left(\begin{array}{cccc}
\mathbf{0} & \mathbf{0} & \cdots & \mathbf{0} \\
\mathbf{V}_{(2,1)} & \mathbf{0} & \cdots & \mathbf{0} \\
\vdots & \vdots & & \vdots \\
\mathbf{V}_{(k, 1)} & \mathbf{V}_{(k, 2)} & \cdots & \mathbf{0}
\end{array}\right) .
$$

Let the gradient with respect to a, $\lambda$ be zero, and we have

$$
\left(\begin{array}{cc}
\mathbf{0} & \mathbf{H}^{\prime} \\
\mathbf{H} & \mathbf{V}_{1}+\mathbf{V}_{3}+\mathbf{V}_{4}
\end{array}\right)\left(\begin{array}{l}
\lambda \\
\mathbf{a}
\end{array}\right)=\left(\begin{array}{c}
\mathbf{h} \\
\mathbf{V}_{2}
\end{array}\right)
$$

After a small amount of algebra, the a solution gives

$$
\begin{aligned}
\mathbf{a}= & \mathbf{Q}^{-1} \mathbf{H}\left(\mathbf{H}^{\prime} \mathbf{Q}^{-1} \mathbf{H}\right)^{-1} \mathbf{h} \\
& +\left[\mathbf{Q}^{-1}-\mathbf{Q}^{-1} \mathbf{H}\left(\mathbf{H}^{\prime} \mathbf{Q}^{-1} \mathbf{H}\right)^{-1} \mathbf{H}^{\prime} \mathbf{Q}^{-1}\right] \mathbf{V}_{2}
\end{aligned}
$$

Note that the parameters $\rho_{i-1}$ are often unknown in (27). We estimate them as follows:

(a) Predict the computer output at $D_{2}=\left(\mathbf{x}_{1}^{(2)}, \ldots, \mathbf{x}_{n_{2}}^{(2)}\right)$, say $\widehat{\mathbf{Z}}_{1}^{(2)}$, based on the assumption $Z_{1}(\cdot) \sim \mathrm{GP}\left(\mathbf{h}^{\prime}(\cdot) \boldsymbol{\beta}_{1}, \sigma_{1}^{2}, b_{1}\right)$ and training data $\left(D_{1}, \mathbf{Z}_{1}\right)$.

(b) Estimate the parameter $\rho_{1}$, say $\hat{\rho}_{1}$, by MLE based on the model $\mathbf{Z}_{2}=\rho_{1} \widehat{\mathbf{Z}}_{1}^{(2)}+\boldsymbol{\delta}_{2}$.

(c) Predict the computer output at $D_{3}=\left(\mathbf{x}_{1}^{(3)}, \ldots, \mathbf{x}_{n_{3}}^{(3)}\right)$, say $\widehat{\mathbf{Z}}_{2}^{(3)}$, based on the model $Z_{2}(\cdot)=\hat{\rho}_{1} Z_{1}(\cdot)+\delta_{2}(\cdot)$ and training data $\left(D_{2}, \mathbf{Z}_{2}\right)$.

(d) Estimate other parameters $\rho_{2}, \ldots, \rho_{k-1}$ iteratively.

By the multivariate normal assumption based on the model $\mathbf{Z}_{i}=\rho_{i-1} \widehat{\mathbf{Z}}_{i-1}^{(i)}+\boldsymbol{\delta}_{i}$, the log likelihood (up to an additive constant) is

$$
\begin{aligned}
&- \frac{1}{2}\left(\mathbf{Z}_{i}-\rho_{i-1} \widehat{\mathbf{Z}}_{i-1}^{(i)}-\mathbf{H}_{i} \boldsymbol{\beta}_{i}\right)^{\prime}\left(\sigma_{i}^{2} \mathbf{A}_{i}\left(D_{i}\right)\right)^{-1} \\
& \cdot\left(\mathbf{Z}_{i}-\rho_{i-1} \widehat{\mathbf{Z}}_{i-1}^{(i)}-\mathbf{H}_{i} \boldsymbol{\beta}_{i}\right),
\end{aligned}
$$


TABLE 1: Input factors in Section 5.

\begin{tabular}{|c|c|c|c|c|}
\hline Factor & $\begin{array}{l}\text { filling velocity } \\
\qquad\left(x_{1}\right)\end{array}$ & $\begin{array}{l}\text { initial pressure } \\
\left(x_{2}\right) \\
\end{array}$ & $\begin{array}{l}\text { increase rate of pressure } \\
\qquad\left(x_{3}\right)\end{array}$ & $\begin{array}{l}\text { initial temperature } \\
\left(x_{4}\right)\end{array}$ \\
\hline Range & {$[30,60]$} & {$[20,40]$} & {$[0.5,9.5]$} & {$[725,745]$} \\
\hline Unit & $\mathrm{mm} / \mathrm{s}$ & Кра & $\mathrm{Kpa} / \mathrm{s}$ & ${ }^{\circ} \mathrm{C}$ \\
\hline
\end{tabular}

and then the MLE of $\rho_{i-1}$ is

$$
\begin{aligned}
\widehat{\rho}_{i-1}= & \mathbf{Q}_{1}^{-1}\left(\widehat{\mathbf{Z}}_{i-1}^{(i)}\right)^{\prime}\left(\mathbf{A}_{i}\left(D_{i}\right)\right)^{-1} \mathbf{Z}_{i} \\
& -\mathbf{Q}_{1}^{-1} \mathbf{A}_{1}^{\prime} \mathbf{B}_{1}^{-1} \mathbf{H}_{i}^{\prime}\left(\mathbf{A}_{i}\left(D_{i}\right)\right)^{-1} \mathbf{Z}_{i} .
\end{aligned}
$$

The empirical BLUP can be obtained by using (29).

\section{BLUPs for Continuous Levels}

For continuous levels, we assume that as the tuning parameter $t$ gets closer to zero, the output of the computer experiment gets closer to the truth-value. Model [5] is

$$
W(\mathbf{x}, t)=V(\mathbf{x})+Z(\mathbf{x}, t)
$$

where $V(\mathbf{x})$ is a stationary Gaussian process with mean function

$$
E[V(\mathbf{x})]=\mathbf{f}_{1}^{\prime}(\mathbf{x}) \boldsymbol{\beta}_{1}
$$

and covariance function

$$
\operatorname{cov}\left(V\left(\mathbf{x}_{1}\right), V\left(\mathbf{x}_{2}\right)\right)=\sigma_{1}^{2} K_{\phi_{1}}\left(\mathbf{x}_{1}, \mathbf{x}_{2}\right),
$$

$Z(\mathbf{x}, t)$ is a nonstationary Gaussian process with mean structure

$$
E[Z(\mathbf{x}, t)]=\mathbf{f}_{2}^{\prime}(\mathbf{x}) \boldsymbol{\beta}_{2}
$$

and covariance structures are

$$
\begin{aligned}
\operatorname{cov} & \left(Z\left(\mathbf{x}_{1}, t_{1}\right), Z\left(\mathbf{x}_{2}, t_{2}\right)\right) \\
= & \sigma_{2}^{2} K_{\phi_{2}}\left(\mathbf{x}_{1}, \mathbf{x}_{2}\right) \min \left(t_{1}, t_{2}\right)^{l},
\end{aligned}
$$

and $V$ and $Z$ are mutually independent. It follows that the covariance function of $W$ has the form

$$
\begin{aligned}
\operatorname{cov} & \left(W\left(\mathbf{x}_{1}, t_{1}\right), W\left(\mathbf{x}_{2}, t_{2}\right)\right) \\
= & \operatorname{cov}\left(V\left(\mathbf{x}_{1}\right), V\left(\mathbf{x}_{2}\right)+\operatorname{cov}\left(Z\left(\mathbf{x}_{1}, t_{1}\right), Z\left(\mathbf{x}_{2}, t_{2}\right)\right)\right. \\
= & \sigma_{1}^{2} K_{\phi_{1}}\left(\mathbf{x}_{1}, \mathbf{x}_{2}\right)+\sigma_{2}^{2} K_{\phi_{2}}\left(\mathbf{x}_{1}, \mathbf{x}_{2}\right) \min \left(t_{1}, t_{2}\right)^{l} .
\end{aligned}
$$

Theorem 3. The BLUP of $W_{0}=W\left(\mathbf{x}_{0}, 0\right)$ based on training data $\mathbf{W}=\left(W\left(\mathbf{x}_{1}, t_{1}\right), \ldots, W\left(\mathbf{x}_{n}, t_{n}\right)\right)^{\prime}$ is $\mathbf{a}^{\prime} \mathbf{W}$, where

$$
\begin{aligned}
\mathbf{a}= & \mathbf{B}^{-1} \mathbf{A}\left(\mathbf{A}^{\prime} \mathbf{B}^{-1} \mathbf{A}\right)^{-1} \mathbf{v} \\
& +\sigma_{1}^{2}\left[\mathbf{B}^{-1}-\mathbf{B}^{-1} \mathbf{A}\left(\mathbf{A}^{\prime} \mathbf{B}^{-1} \mathbf{A}\right)^{-1} \mathbf{A}^{\prime} \mathbf{B}^{-1}\right] \mathbf{r}_{0}
\end{aligned}
$$

where the notation is given in the proof.
Proof. Since the linear predictor $\mathbf{a}^{\prime} \mathbf{W}+C$ is unbiased for $W_{0}$,

$$
\begin{aligned}
\sum_{i=1}^{n} \mathbf{a}_{i}^{\prime} \mathbf{f}_{1}^{\prime}\left(\mathbf{x}_{i}\right) & =\mathbf{f}_{1}^{\prime}\left(\mathbf{x}_{0}\right), \\
\sum_{i=1}^{n} \mathbf{a}_{i}^{\prime} \mathbf{f}_{2}^{\prime}\left(\mathbf{x}_{i}\right) & =\mathbf{0}, \\
C & =0
\end{aligned}
$$

The Lagrange function is

$$
\begin{aligned}
L= & \sigma_{1}^{2} \mathbf{a}^{\prime} \mathbf{R}_{1} \mathbf{a}-2 \sigma_{1}^{2} \mathbf{a}^{\prime} \mathbf{r}_{0}+\sigma_{1}^{2}+\sigma_{2}^{2} \mathbf{a}^{\prime} \mathbf{R}_{2} \mathbf{a} \\
& +2 \lambda_{1}^{\prime}\left[\mathbf{F}_{1}^{\prime} \mathbf{a}-\mathbf{f}_{0}\right]+2 \lambda_{2}^{\prime} \mathbf{F}_{2}^{\prime} \mathbf{a}
\end{aligned}
$$

where $\mathbf{F}_{i}=\left(\mathbf{f}_{i}\left(\mathbf{x}_{1}\right), \mathbf{f}_{i}\left(\mathbf{x}_{2}\right), \ldots, \mathbf{f}_{i}\left(\mathbf{x}_{n}\right)\right)^{\prime}, i=1,2 ; \mathbf{R}_{1}=\left(K_{\phi_{1}}\left(\mathbf{x}_{i}\right.\right.$, $\left.\left.\mathbf{x}_{j}\right)\right), \mathbf{r}_{0}=\left(K_{\phi_{1}}\left(\mathbf{x}_{i}, \mathbf{x}_{0}\right)\right)$, and $\mathbf{R}_{2}=\left(K_{\phi_{2}}\left(\mathbf{x}_{i}, \mathbf{x}_{j}\right) \min \left(t_{i}, t_{j}\right)^{l}\right), 1 \leq$ $i, j \leq n$. Let the gradient with respect to $\mathbf{a}, \lambda_{1}, \lambda_{2}$ be zero, and we have

$$
\left(\begin{array}{ccc}
\mathbf{0} & \mathbf{0} & \mathbf{F}_{1}^{\prime} \\
\mathbf{0} & \mathbf{0} & \mathbf{F}_{2}^{\prime} \\
\mathbf{F}_{1} & \mathbf{F}_{2} & \sigma_{1}^{2} \mathbf{R}_{1}+\sigma_{2}^{2} \mathbf{R}_{2}
\end{array}\right)\left(\begin{array}{c}
\lambda_{1} \\
\boldsymbol{\lambda}_{2} \\
\mathbf{a}
\end{array}\right)=\left(\begin{array}{c}
\mathbf{f}_{0} \\
\mathbf{0} \\
\sigma_{1}^{2} \mathbf{r}_{0}
\end{array}\right)
$$

After a small amount of algebra, the a solution gives

$$
\begin{aligned}
\mathbf{a}= & \mathbf{B}^{-1} \mathbf{A}\left(\mathbf{A}^{\prime} \mathbf{B}^{-1} \mathbf{A}\right)^{-1} \mathbf{v} \\
& +\sigma_{1}^{2}\left[\mathbf{B}^{-1}-\mathbf{B}^{-1} \mathbf{A}\left(\mathbf{A}^{\prime} \mathbf{B}^{-1} \mathbf{A}\right)^{-1} \mathbf{A}^{\prime} \mathbf{B}^{-1}\right] \mathbf{r}_{0}
\end{aligned}
$$

where $\mathbf{A}=\left(\mathbf{F}_{1}, \mathbf{F}_{2}\right), \mathbf{B}=\sigma_{1}^{2} \mathbf{R}_{1}+\sigma_{2}^{2} \mathbf{R}_{2}$, and $\mathbf{v}=\left(\mathbf{f}_{0}^{\prime}, \mathbf{0}\right)^{\prime}$. This completes the proof.

\section{A Real Application}

Casting is one of the most important manufacturing processes for making metal products. Nowadays computer simulations for casting processes are commonly used. In the project we consider a low-pressure die-casting process that produces a certain component of satellites and study how the process parameters influence the temperature difference at a key location on the casting product. In the simulations the input factors are four-process parameters shown in Table 1. We conduct the experiment with two levels of accuracy and specify the run sizes of the high-accuracy and low-accuracy simulations as 16 and 32, respectively. The design and its corresponding responses are showed in Table 2. We then use the BLUP (5) to predict the high-accuracy responses at the points where we have only low-accuracy responses. The results are also reported in Table 2; see asterisked values. It can be seen that the prediction results are satisfactory. 


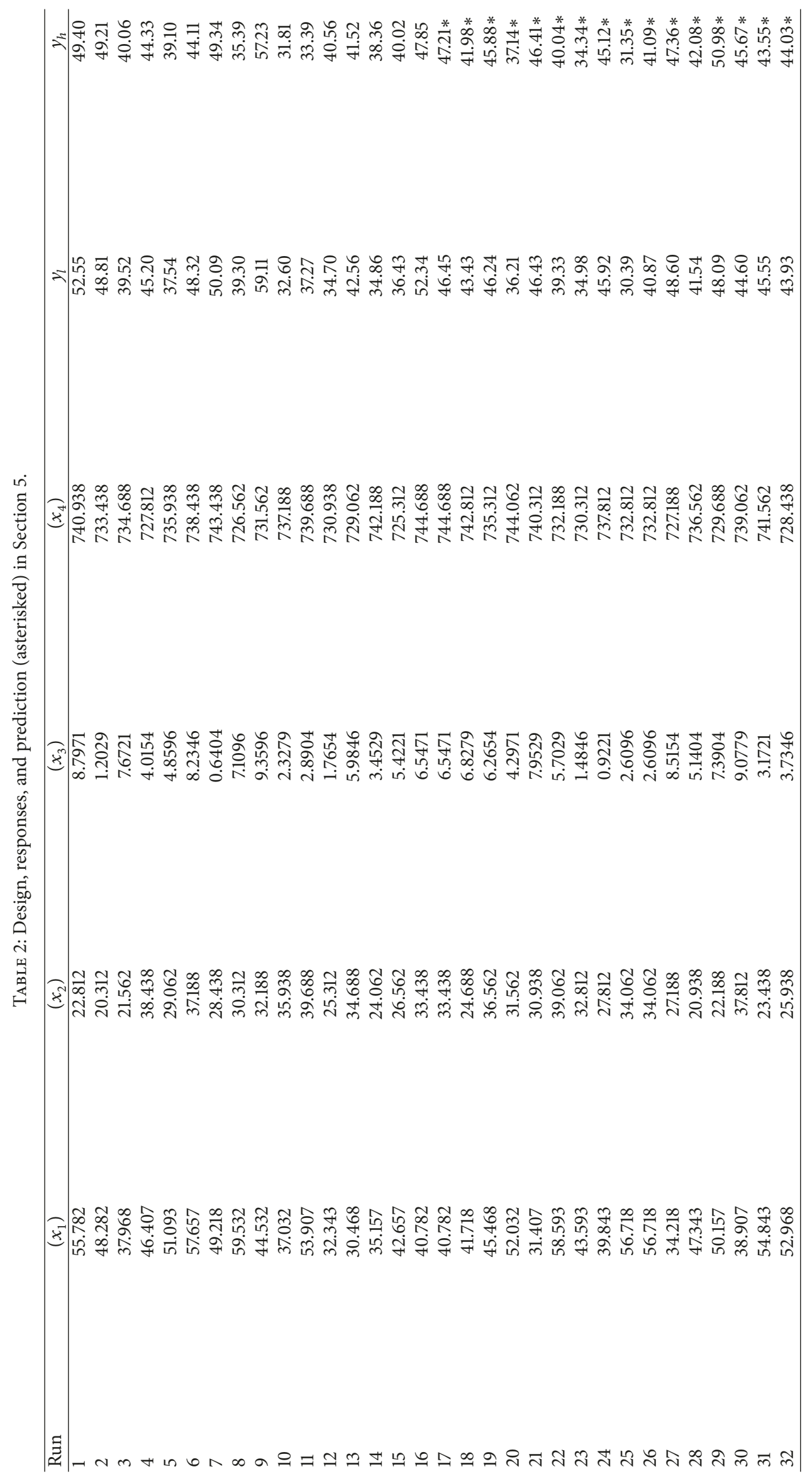




\section{Concluding Remarks}

We have provided computable BLUPs for three classes of Gaussian process models, which are commonly used in modeling multifidelity computer experiments. A real engineering application is presented to show the effectiveness of our method. An advantage of our method is its explicit forms. The predictors by our method are consistent (or close to) with Bayesian predictors in the literature. Furthermore, our method does not require nested structure of the design $[6,7]$. A limitation of our method lies in that its expression involves some unknown parameters which we need to estimate in advance. The MLE method or modified MLE methods can be used to solve this problem. Our method to derive BLUPs can also be used for other related cases, including sequential settings [8], computer experiments with both qualitative and quantitative factors [9], and sensitivity analysis [10].

\section{Conflicts of Interest}

There are not any conflicts of interest related to this paper.

\section{Acknowledgments}

This work is supported by funding from Chinese Ministry of Science and Technology (Grant no. 2016YFF0203801), the National Natural Science Foundation of China (Grant nos. 11601027,11671386 , and 11471172), and research funding from BUCEA (Grant nos. 21082716014, 00331616031).

\section{References}

[1] T. J. Santner, B. J. Williams, and W. I. Notz, The Design And Analysis of Computer Experiments, Springer, New York, NY, USA, 2003.

[2] M. C. Kennedy and A. O'Hagan, "Predicting the output from a complex computer code when fast approximations are available," Biometrika, vol. 87, no. 1, pp. 1-13, 2000.

[3] P. Z. G. Qian, C. C. Seepersd, V. R. Joseph, J. K. Allen, and C. F. J. Wu, "Building surrogate models based on detailed and approximate simulations," Journal of Mechanical Design, vol. 128, pp. 668-677, 2006.

[4] P. Z. G. Qian and C. F. J. Wu, "Bayesian hierarchical modelling for integrating low-accuracy and high-accuracy experiments," Technometrics, vol. 50, no. 2, pp. 192-204, 2008.

[5] R. Tuo, C. F. Wu, and D. Yu, "Surrogate modeling of computer experiments with different mesh densities," Technometrics. A Journal of Statistics for the Physical, Chemical and Engineering Sciences, vol. 56, no. 3, pp. 372-380, 2014.

[6] P. Z. Qian, "Nested Latin hypercube designs," Biometrika, vol. 96, no. 4, pp. 957-970, 2009.

[7] D. Chen and S. Xiong, "Flexible nested Latin hypercube designs for computer experiments," Journal of Quality Technology, vol. 49, no. 4, pp. 337-353, 2017.

[8] S. Xiong, P. Z. Qian, and C. F. Wu, "Sequential design and analysis of high-accuracy and low-accuracy computer codes," Technometrics. A Journal of Statistics for the Physical, Chemical and Engineering Sciences, vol. 55, no. 1, pp. 37-46, 2013.

[9] P. Z. Qian, H. Wu, and C. F. Wu, "Gaussian process models for computer experiments with qualitative and quantitative factors," Technometrics. A Journal of Statistics for the Physical, Chemical and Engineering Sciences, vol. 50, no. 3, pp. 383-396, 2008.

[10] S. Xiong, X. He, Y. He, and W. Mu, "Sensitivity analysis using permutations," Statistica Sinica, vol. 28, pp. 817-837, 2018. 


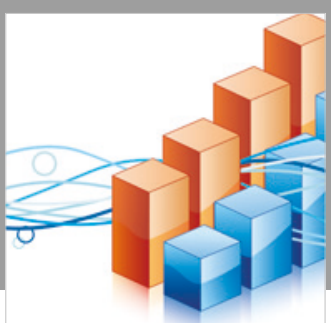

Advances in

Operations Research

\section{-n-m}
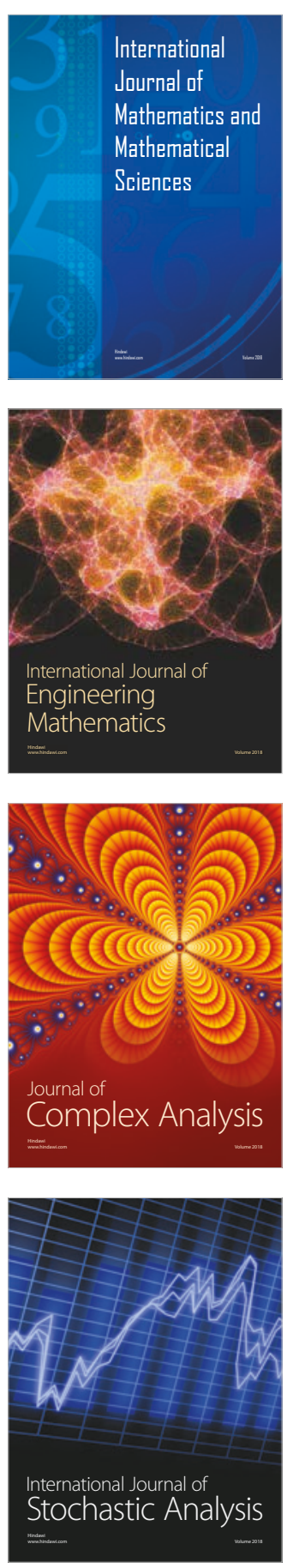
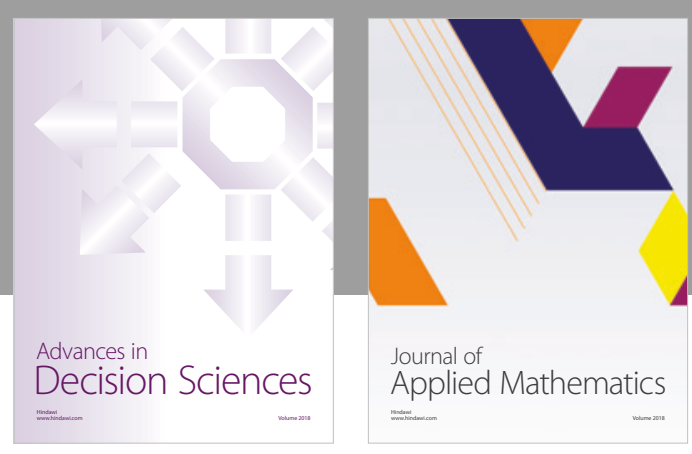

Journal of

Applied Mathematics
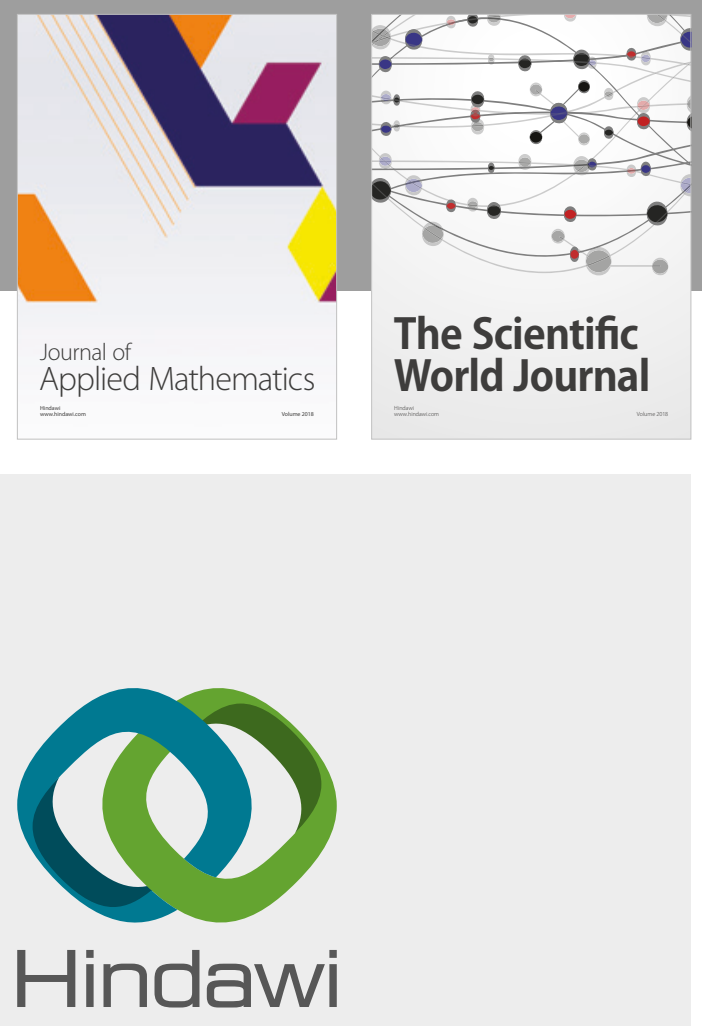

Submit your manuscripts at

www.hindawi.com

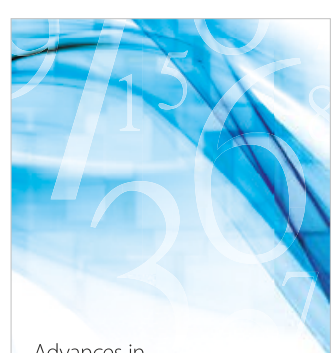

Advances in
Numerical Analysis
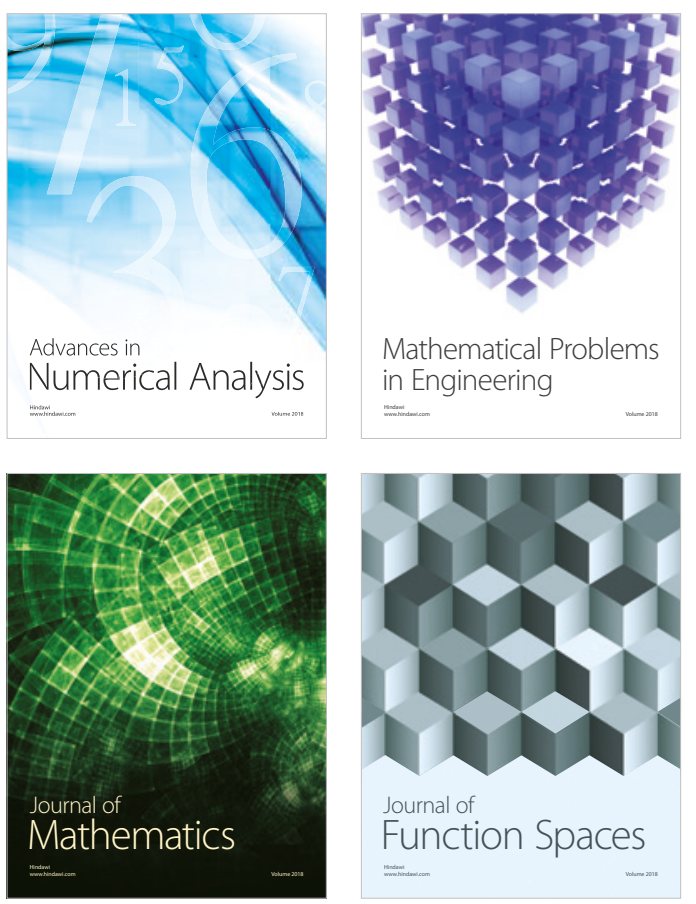

Mathematical Problems in Engineering

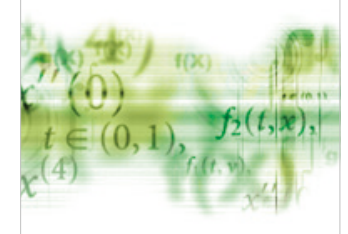

International Journal of

Differential Equations

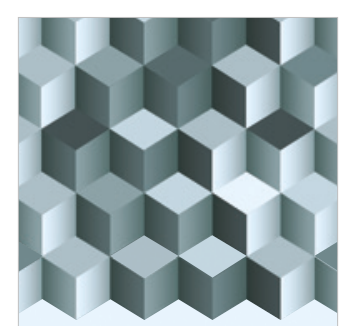

Journal of

Function Spaces

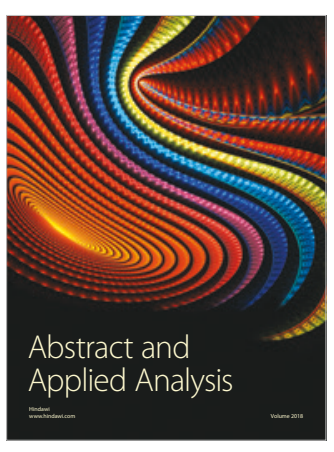

The Scientific

World Journal

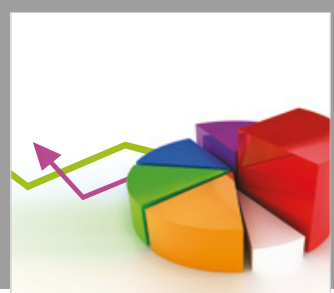

Journal of

Probability and Statistics
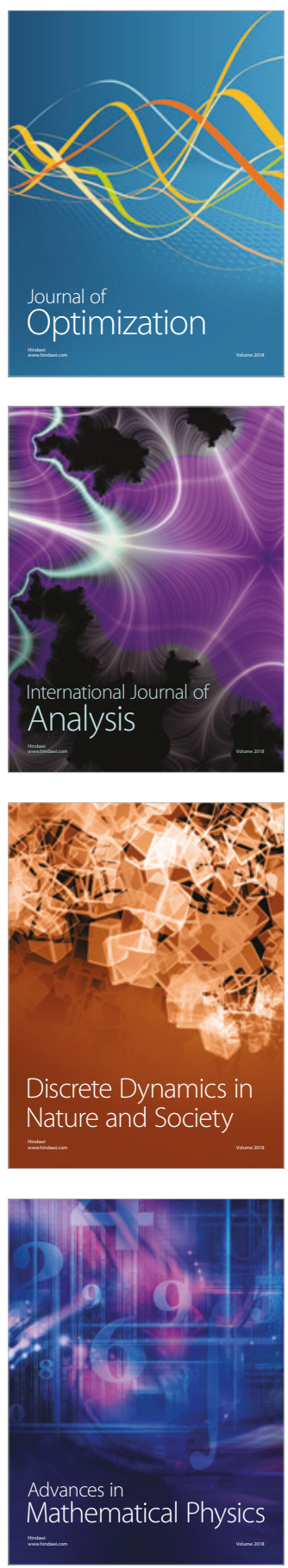